\title{
EXCHANGE RINGS WITH MANY UNITS
}

\author{
HuAnyin Chen \\ Hangzhou Normal University, China
}

\begin{abstract}
A ring $R$ satisfies Goodearl-Menal condition provided that for any $x, y \in R$, there exists a $u \in U(R)$ such that $x-u, y-u^{-1} \in U(R)$. If $R / J(R)$ is an exchange ring with primitive factors artinian, then $R$ satisfies Goodearl-Menal condition if, and only if it has no homomorphic images $\mathbb{Z} / 2 \mathbb{Z}, \mathbb{Z} / 3 \mathbb{Z}, M_{2}(\mathbb{Z} / 2 \mathbb{Z})$. Exchange rings satisfying the primitive criterion are also studied.
\end{abstract}

\section{INTRODUCTION}

A ring $R$ is said to have unit 1-stable range if $a R+b R=R$ implies there exists a $u \in U(R)$ such that $a+b u \in U(R)$, where $U(R)$ denotes the group of all invertible elements in $R$. If $R$ has unit 1-stable range, then $K_{1}(R) \cong U(R) / V(R)$, where $V(R)=\left\{(1+a b)(1+b a)^{-1} \mid 1+a b \in U(R)\right\}$ (cf. [9, Theorem 1.2]). Also we note that $K_{2}(R)$ is generated by $\langle a, b, c\rangle_{*}$ if $R$ is a commutative ring having unit 1-stable range (cf. [11]). In [6], Goodearl and Menal introduced a simple condition:

For any $x, y \in R$, there exists a $u \in U(R)$ such that $x-u, y-u^{-1} \in U(R)$.

They discovered that this condition supplied for many classes of rings having unit 1-stable range. As is well known, such condition coincides with unit 1-stable range for any unital complex $C^{*}$-algebra (see [6, Theorem 4.1]). This condition was also investigated in [3-6]. We say that a ring $R$ satisfies Goodearl-Menal condition provided that such condition holds. In particular, Goodefroid observed that any topological ring $R$ for which the group of units is open and dense in $R$ satisfies Goodearl-Menal condition. If $R$ satisfies Goodearl-Menal condition, by [9, Theorem 1.2 and Theorem

2010 Mathematics Subject Classification. 16E50, 16U99.

Key words and phrases. Goodearl-Menal condition, exchange ring, semilocal ring. 
1.3], the natural map $U(R)^{a b} \rightarrow K_{1}(R)$ is an isomorphism. Furthermore, $U(R)^{a b} \cong G L_{n}(R) / E_{n}(R)$ for any $n \geq 2$ (see [6, Theorem 1.4]).

A ring $R$ is said to be an exchange ring provided that for any $a \in R$, there exists an idempotent $e \in R a$ such that $1-e \in R(1-a)$. The class of exchange rings is very large. It includes regular rings, $\pi$-regular rings, strongly $\pi$-regular rings, semiperfect rings, left or right continuous rings, clean rings, and unit $C^{*}$-algebras of real rank zero. Such rings have been extensively studied by many authors (cf. [1-2], [7], [10] and [13-14]). For general theory of exchange rings, we refer the reader to [12]. In [13, Theorem 1], $\mathrm{Yu}$ proved that every exchange ring with artinian primitive factors has stable range one. If $R / J(R)$ is an exchange ring with primitive factors artinian, we prove that $R$ satisfies Goodearl-Menal condition if, and only if it has no homomorphic images $\mathbb{Z} / 2 \mathbb{Z}, \mathbb{Z} / 3 \mathbb{Z}, M_{2}(\mathbb{Z} / 2 \mathbb{Z})$. Exchange rings satisfying the primitive criterion are also studied.

Throughout, all rings are associative with an identity and all right $R$ modules are unital. $M_{n}(R)$ denotes the ring of all $n \times n$ matrices over $R$, $G L_{n}(R)$ denotes the $n$-dimensional general linear group of $R$. We use $|S|$ to stands for the cardinal number of the set $S$.

\section{DiVISION RINGS}

In this section, we investigate Goodeal-Menal condition for the matrix rings over a division ring, which will be used in the sequel. A Morita context $(A, B, M, N, \psi, \phi)$ consists of two rings $A, B$, two bimodules ${ }_{A} N_{B},_{B} M_{A}$ and a pair of bimodule homomorphisms $\psi: N \otimes M \rightarrow A$ and $\phi: M \otimes N \rightarrow B$ which satisfy the following associativity: $\psi(n \otimes m) n^{\prime}=n \phi\left(m \otimes{ }^{A} n^{\prime}\right)$ and $\phi(m \bigotimes n) m^{\prime}=m \psi\left(n \otimes m^{\prime}\right)$ for any $m, m^{\prime} \in M, n, n^{\prime} \in N$. These conditions insure that the set $T$ of generalized matrices

$$
\left(\begin{array}{cc}
a & n \\
m & b
\end{array}\right) \quad a \in A, b \in B, m \in M, n \in N
$$

will form a ring, called the ring of the Morita context. The class of the rings of Morita contexts includes all $2 \times 2$ matrix rings and all triangular matrix rings. We start by the following elementary result.

Lemma 2.1. If $A$ and $B$ satisfy Goodearl-Menal condition, then so does $T$.

ProOF. Let

$$
\left(\begin{array}{cc}
a_{1} & n_{1} \\
m_{1} & b_{1}
\end{array}\right),\left(\begin{array}{cc}
a_{2} & n_{2} \\
m_{2} & b_{2}
\end{array}\right) \in T .
$$

Then there exist some $a \in U(A)$ and $b \in U(B)$ such that $a_{1}-a=u_{1} \in$ $U(A), 1_{A}-a_{2} a=v_{1} \in U(A),\left(b_{1}-\phi\left(m_{1} u_{1}^{-1} \otimes n_{1}\right)\right)-b=u_{2} \in U(B)$ and 
$1_{B}-\left(\phi\left(m_{2} a v_{1}^{-1} \otimes n_{2}\right)+b_{2}\right) b=v_{2} \in U(B)$. One easily checks that

$$
\begin{aligned}
& \left(\begin{array}{cc}
a_{1} & n_{1} \\
m_{1} & b_{1}
\end{array}\right)-\left(\begin{array}{cc}
a & 0 \\
0 & b
\end{array}\right) \\
& \quad=\left(\begin{array}{cc}
u_{1}^{-1}+u_{1}^{-1} \psi\left(n_{1} u_{2}^{-1} \bigotimes m_{1} u_{1}^{-1}\right) & -u_{1}^{-1} n_{1} u_{2}^{-1} \\
-u_{2}^{-1} m_{1} u_{1}^{-1} & u_{2}^{-1}
\end{array}\right)^{-1}
\end{aligned}
$$

and

$$
\begin{aligned}
1_{T}- & \left(\begin{array}{cc}
a_{2} & n_{2} \\
m_{2} & b_{2}
\end{array}\right)\left(\begin{array}{cc}
a & 0 \\
0 & b
\end{array}\right) \\
& =\left(\begin{array}{cc}
v_{1}^{-1}+\psi\left(-v_{1}^{-1} n_{2} b v_{2}^{-1} \bigotimes-m_{2} a v_{1}^{-1}\right) & -v_{1}^{-1} n_{2} b v_{2}^{-1} \\
-v_{2}^{-1} m_{2} a v_{1}^{-1} & v_{2}^{-1}
\end{array}\right)^{-1},
\end{aligned}
$$

and therefore we complete the proof.

Theorem 2.2. Let $A$ and $B$ be right $R$-modules. If $\operatorname{End}_{R}(A)$ and $\operatorname{End}_{R}(B)$ satisfy Goodearl-Menal condition, then so does $\operatorname{End}_{R}(A \oplus B)$.

Proof. Let $e: A \oplus B \rightarrow A \oplus B$ given by $e(a+b)=a$ for any $a \in$ $A, b \in B$. Then $e \operatorname{End}_{R}(A \oplus B) e \cong \operatorname{End}_{R}(e(A \oplus B)) \cong \operatorname{End}_{R}(A)$. Likewise, $\left(1_{A \oplus B}-e\right) \operatorname{End}_{R}(A \oplus B)\left(1_{A \oplus B}-e\right) \cong \operatorname{End}_{R}(B)$. As is well known, the endomorphisms of the direct sum are given by a suitable Morita context. Thus, we get

$$
\operatorname{End}_{R}(A \oplus B) \cong\left(\begin{array}{cc}
e \operatorname{End}_{R}(A \oplus B) e & e \operatorname{End}_{R}(A \oplus B)(1-e) \\
(1-e) \operatorname{End}_{R}(A \oplus B) e & (1-e) \operatorname{End}_{R}(A \oplus B)(1-e)
\end{array}\right) .
$$

By hypothesis and Lemma 2.1, $\operatorname{End}_{R}(A \oplus B)$ satisfies Goodearl-Menal condition.

Let $e \in R$ be an idempotent. If $e R e$ and $(1-e) R(1-e)$ satisfy GoodearlMenal condition, it follows from Theorem 2.2 that $R$ satisfies Goodearl-Menal condition. The converse is not true. For instance, choosing $R=M_{3}\left(\mathbb{Z}_{2}\right)$, and $e=\operatorname{diag}(1,0,0)$. Then $R$ satisfies Goodeal-Menal condition, but $e R e \cong \mathbb{Z}_{2}$ does not satisfy such condition.

Corollary 2.3. A ring $R$ satisfies Goodearl-Menal condition if, and only if so does the ring $T M_{n}(R)$ of all $n \times n$ upper triangular matrix over $R$.

Proof. $\Leftarrow$ : This is obvious.

$\Rightarrow$ : By Theorem 2.2 and induction, we complete the proof.

A ring $R$ is unit-regular provided that for any $x \in R$, there exists a $u \in U(R)$ such that $x=x u x$, e.g., every division ring and the endomorphism ring of any finite-dimensional vector space over a division ring. 
Lemma 2.4. Let $R$ be a unit-regular ring, and let $n \in \mathbb{N}$. Then $M_{n}(R)$ satisfies Goodearl-Menal condition if, and only if for any $X \in M_{n}(R)$ and diagonal matrix $Y \in M_{n}(R)$, there exists a $U \in G L_{n}(R)$ such that $X-U, Y-$ $U^{-1} \in G L_{n}(R)$.

Proof. $\Rightarrow$ : This is an instance of the definition.

$\Leftarrow$ : For any $X, Y \in M_{n}(R)$, there exist $U, V \in G L_{n}(R)$ such that $U X V=$ $\operatorname{diag}\left(x_{1}, \cdots, x_{n}\right)$ for some $x_{1}, \cdots, x_{n} \in R$. By hypothesis, we have some $W \in G L_{n}(R)$ such that $\operatorname{diag}\left(x_{1}, \cdots, x_{n}\right)-W, V Y U-W^{-1} \in G L_{n}(R)$. Thus, $A-U^{-1} W V^{-1}, Y-\left(U^{-1} W V^{-1}\right)^{-1} \in G L_{n}(R)$, as required.

It is directly verified that $\mathbb{Z} / 2 \mathbb{Z}$ and $\mathbb{Z} / 3 \mathbb{Z}$ do not satisfy Goodearl-Menal condition. Choose

$$
A=\left(\begin{array}{ll}
0 & 0 \\
1 & 0
\end{array}\right), B=\left(\begin{array}{ll}
0 & 0 \\
0 & 1
\end{array}\right) \in M_{2}(\mathbb{Z} / 2 \mathbb{Z}) .
$$

For any $U \in G L_{2}(\mathbb{Z} / 2 \mathbb{Z})$, we can check that $A-U \notin G L_{2}(\mathbb{Z} / 2 \mathbb{Z})$ or $B-U^{-1} \notin$ $G L_{2}(\mathbb{Z} / 2 \mathbb{Z})$. Thus, $M_{2}(\mathbb{Z} / 2 \mathbb{Z})$ does not satisfy Goodearl-Menal condition. It is worth noting that the Goodearl-Menal condition is obviously preserved in homomorphic images.

Proposition 2.5. Let $D$ be a division ring. Then $M_{n}(D)$ satisfies Goodearl-Menal condition if $n=1, D \neq \mathbb{Z} / 2 \mathbb{Z}, \mathbb{Z} / 3 \mathbb{Z}$; or $n=2, D \neq \mathbb{Z} / 2 \mathbb{Z}$; or $n \geq 3$.

Proof. It is proved by a computer in Microsoft Visual $\mathrm{C}++$ that $M_{3}(\mathbb{Z} / 2 \mathbb{Z}), M_{4}(\mathbb{Z} / 2 \mathbb{Z}), M_{5}(\mathbb{Z} / 2 \mathbb{Z}), M_{2}(\mathbb{Z} / 3 \mathbb{Z})$ and $M_{3}(\mathbb{Z} / 3 \mathbb{Z})$ satisfy Goodearl-Menal condition.

Let $n \geq 2$. In view of Theorem $2.2, M_{3 n}(\mathbb{Z} / 2 \mathbb{Z})$ and $M_{3(n-1)}(\mathbb{Z} / 2 \mathbb{Z})$ satisfy Goodearl-Menal condition. Clearly, we see that $3 n+1=3(n-$ $1)+4,3 n+2=3(n-1)+5$. According to Theorem $2.2, M_{3 n+1}(\mathbb{Z} / 2 \mathbb{Z})$ and $M_{3 n+2}(\mathbb{Z} / 2 \mathbb{Z})(n \in \mathbb{N})$ satisfy Goodearl-Menal condition. Consequently, $M_{n}(\mathbb{Z} / 2 \mathbb{Z})(n \geq 3)$ satisfies Goodearl-Menal condition.

By virtue of Theorem 2.2, $M_{2 n}(\mathbb{Z} / 3 \mathbb{Z})$ satisfies Goodearl-Menal condition. Since $2 n+1=2(n-1)+3$, analogously, $M_{2 n+1}(\mathbb{Z} / 3 \mathbb{Z})$ satisfies GoodearlMenal condition. Thus, $M_{n}(\mathbb{Z} / 3 \mathbb{Z})(n \geq 2)$ satisfies Goodearl-Menal condition.

One easily checks that every division ring with at least 4 elements satisfies Goodearl-Menal condition. Therefore we complete the proof by Theorem 2.2.

\section{EXCHANGE RINGS WITH PRIMITIVE FACTORS ARTINIAN}

Lemma 3.1. Let $R$ be a ring. Then $R$ satisfies Goodearl-Menal condition if, and only if for any $x, y \in R$, there exists a $u \in U(R)$ such that $(x-u)(y u-$ 1) $\in U(R)$. 
PROOF. $\Rightarrow$ : It is clear.

$\Leftarrow$ : Assume that $a b=1$. Then there exists a $u \in U(R)$ such that $(b-$ $u)(a u-1)=1$. Write $v=b-u$ and $w=a-u^{-1}$. Then $v w u=1$ and $a v=a(b-u)=1-a u=-w u$, and so $a=-w u w u$. Thus,

$$
b a=\left(-v^{2}\right)(-w u w u) b a=\left(-v^{2}\right)(a b)(-w u w u)=\left(-v^{2}\right)(-w u w u)=1 .
$$

That is, $R$ is directly finite, as required.

THEOREM 3.2. Let $R / J(R)$ be an exchange ring whose primitive factors are artinian. Then $R$ satisfies Goodearl-Menal condition if, and only if it does not admit $\mathbb{Z} / 2 \mathbb{Z}, \mathbb{Z} / 3 \mathbb{Z}, M_{2}(\mathbb{Z} / 2 \mathbb{Z})$ as homomorphic images.

Proof. One direction is obvious by the observation on quotients of rings with Goodearl-Menal condition.

Conversely, letting $S=R / J(R)$, assume that there exist some $x, y \in S$ such that $(x-u)(y u-1) \notin U(S)$ for any $u \in U(S)$. Let $\Omega$ be the set of all ideals $I$ of $S$ such that $(x-u)(y u-1)$ is not a unit modulo $I$ for any $u+I \in U(S / I)$. Clearly, $\Omega \neq \emptyset$. Choose an ascending chain $A_{1} \subseteq A_{2} \subseteq \cdots \subseteq A_{n} \subseteq \cdots$ in $\Omega$. Set $M=\bigcup_{i=1}^{\infty} A_{i}$. Then $M$ is an ideal of $S$. Assume that $M$ is not in $\Omega$. We have $u+M \in U(S / M)$ such that $(x-u)(y u-1)+M \in U(S / M)$. So there are positive integers $n_{i}(1 \leq i \leq 4)$ such that

$$
\begin{aligned}
& (x-u)(y u-1) s-1 \in A_{n_{1}}, \quad s(x-u)(y u-1)-1 \in A_{n_{2}}, \\
& u t-1 \in A_{n_{3}} \text { and } t u-1 \in A_{n_{4}}
\end{aligned}
$$

for some $s, t \in S$. Let $n=\max \left\{n_{1}, n_{2}, n_{3}, n_{4}\right\}$. Then $\overline{(x-u)(y u-1)} \in$ $U\left(S / A_{n}\right)$ for $u+A_{n} \in U\left(S / A_{n}\right)$, a contradiction. This implies that $M \in \Omega$. By using Zorn's Lemma, there exists an ideal $Q$ of $S$ such that it is maximal in $\Omega$.

Set $T=S / Q$. If $J(T) \neq 0$, then $J(T)=K / Q$ for some $K \supsetneqq Q$. Clearly, $T / J(T) \cong S / K$. By the maximality of $Q$, there is some $(v+Q)+J(T) \in$ $U(T / J(T))$ such that

$$
((x-v)(y v-1)+Q)+J(T) \in U(T / J(T)) .
$$

Clearly, $v+Q \in U(S / Q)$. Further, we see that $(x-v)(y v-1)+Q \in U(T)$. This gives a contradiction, and so $J(S / Q)=0$.

Moreover, $S / Q$ is an indecomposable ring. In view of [14, Lemma 3.7], $S / Q \cong M_{n}(D)$ for a division ring $D$. Since $S$ has no isomorphic images $\mathbb{Z} / 2 \mathbb{Z}, \mathbb{Z} / 3 \mathbb{Z}, M_{2}(\mathbb{Z} / 2 \mathbb{Z})$, we have that $|D|=2, n \geq 3$ or $|D|=3, n \geq 2$ or $|D| \geq 4$. In view of Proposition 2.5, $S / Q$ satisfies Goodearl-Menal condition. Thus, we have $w+Q \in U(S / Q)$ such that $\overline{(x-w)(y w-1)} \in U(S / Q)$, a contradiction. According to Lemma 3.1, $S$ satisfies Goodearl-Menal condition. For any $x, y \in R$, we can find some $\bar{w} \in R / J(R)$ such that $\bar{x}-\bar{u}, \bar{x}-\bar{u}^{-1} \in$ 
$U(R / J(R))$. Clearly, $u \in U(R)$. Further, $x-u, y-u^{-1} \in U(R)$. Therefore $R$ satisfies Goodearl-Menal condition.

A ring $R$ is said to be strongly $\pi$-regular provided that for any $x \in R$, there exists some $n \in \mathbb{N}$ such that $x^{n} \in x^{n+1} R$.

COROLlary 3.3. Let $R / J(R)$ be a strongly $\pi$-regular ring whose primitive factors are artinian. Then $R$ satisfies Goodearl-Menal condition if, and only if it does not admit $\mathbb{Z} / 2 \mathbb{Z}, \mathbb{Z} / 3 \mathbb{Z}, M_{2}(\mathbb{Z} / 2 \mathbb{Z})$ as homomorphic images.

Proof. Clearly, $R / J(R)$ is an exchange ring, and so the result follows by Theorem 3.2.

Recall that a ring $R$ is semilocal provided that $R / J(R)$ is artinian. Let $R=\left\{\frac{m}{n} \mid 2,3 \nmid n,(m, n)=1, m, n \in \mathbb{Z}\right\}$. Then $R$ is semilocal with only two maximal ideals $2 R$ and $3 R$. In this case, $R / J(R)$ an exchange ring whose primitive factors are artinian. But $R$ is not an exchange ring. In fact, $R$ has only two idempotents, but $R / J(R) \cong \mathbb{Z}_{2} \oplus \mathbb{Z}_{3}$ has four idempotents, and so idempotents can not be lifted modulo $J(R)$.

Corollary 3.4. Let $R$ be a semilocal ring. Then $R$ satisfies GoodearlMenal condition if, and only if it does not admit $\mathbb{Z} / 2 \mathbb{Z}, \mathbb{Z} / 3 \mathbb{Z}, M_{2}(\mathbb{Z} / 2 \mathbb{Z})$ as homomorphic images.

Proof. Since $R$ is semilocal, $R / J(R)$ is artinian. Thus, $R / J(R)$ is an exchange ring with all primitive factors artinian. Therefore we complete the proof by Theorem 3.2.

Corollary 3.5. Let $A$ be an artinian right $R$-module. If $\frac{1}{2}, \frac{1}{3} \in R$, then $\operatorname{End}_{R}(A)$ satisfies Goodearl-Menal condition.

Proof. Let $S=\operatorname{End}_{R}(A)$. Then $S$ is semilocal, by the Camps-Dicks theorem. Construct an $R$-morphism $\varphi: A \rightarrow A$ given by $\varphi(a)=a \cdot \frac{1}{2}$ for any $a \in A$. Then $\varphi \in A u t_{R}(A)$, and so $\frac{1}{2} \in S$. Likewise, $\frac{1}{3} \in S$. If there exists an ideal $I$ of $S$ such that $S / I \cong \mathbb{Z} / 2 \mathbb{Z}$ or $\mathbb{Z} / 3 \mathbb{Z}$ or $M_{2}(\mathbb{Z} / 2 \mathbb{Z})$, then $\frac{1}{2}, \frac{1}{3} \in S / I$. This gives a contradiction. In view of Corollary $3.4, \operatorname{End}_{R}(A)$ satisfies Goodearl-Menal condition.

Recall that a ring $R$ is of bounded index provided that there exists $n \in \mathbb{N}$ such that $x^{n}=0$ for any nilpotent $x \in R$.

Corollary 3.6. Let $R / J(R)$ be an exchange ring of bounded index. Then $R$ satisfies Goodearl-Menal condition if, and only if it does not admit $\mathbb{Z} / 2 \mathbb{Z}, \mathbb{Z} / 3 \mathbb{Z}, M_{2}(\mathbb{Z} / 2 \mathbb{Z})$ as homomorphic images.

Proof. By virtue of [13, Theorem 3], $R / J(R)$ is an exchange ring with primitive factors artinian. Thus, we obtain the result from Theorem 3.2. 
Example 3.7. Let $R=k[x] /\left(x^{2}\right)=\left\{a+b t \mid a, b \in k, t^{2}=0\right\}$ where $k$ is a field of characteristic 5. Suppose $a+b t \in R$. If $a \neq 0$, then $(a+b t)^{5}=$ $(a+b t)^{6}(a-b t) a^{-2}$. If $a=0$, then $(a+b t)^{2}=(a+b t)^{3}$. Therefore $R$ is a strongly $\pi$-regular ring. Assume that $(a+b t)^{n}=0$ in $R$. Then $(a+b t)^{5 n}=0$, hence $a^{5 n}=\left((a+b t)^{5}\right)^{n}=0$. So $a=0$, and then $(a+b t)^{5}=a^{5}=0$. That is, $R$ is a strongly $\pi$-regular ring of bounded index 5 . Clearly, $\frac{1}{3 !} \in R$. Hence, $R$ is an exchange ring of bounded index. In addition, it has no homomorphic images $\mathbb{Z} / 2 \mathbb{Z}, \mathbb{Z} / 3 \mathbb{Z}, M_{2}(\mathbb{Z} / 2 \mathbb{Z})$. In view of Corollary 3.6, $R$ satisfies GoodearlMenal condition.

A ring $R$ is a right (left) quasi-duo if every maximal right (left) ideal is a two-sided ideal.

COROLlaRY 3.8. Let $R$ be a right (left) quasi-duo exchange ring. Then $R$ satisfies Goodearl-Menal condition if, and only if it does not admit $\mathbb{Z} / 2 \mathbb{Z}$ and $\mathbb{Z} / 3 \mathbb{Z}$ as homomorphic images.

Proof. $\Rightarrow$ : In this case, $R / J(R)$ is abelian, and so it is clear as in the proof of Theorem 3.2.

$\Leftarrow$ : Since $R$ is a right (left) quasi-duo exchange ring, $R / J(R)$ is an exchange ring with all idempotents central. Similarly to [13, Theorem 6], $R / J(R)$ is an exchange ring of bounded index 1. By virtue of Corollary 3.6, $R$ satisfies Goodearl-Menal condition.

Let $R / J(R)$ be an exchange ring with all idempotents central. Analogously, we deduce that $R$ satisfies Goodearl-Menal condition if, and only if it does not admit $\mathbb{Z} / 2 \mathbb{Z}$ and $\mathbb{Z} / 3 \mathbb{Z}$ as homomorphic images.

Proposition 3.9. Let $R / J(R)$ be an exchange ring whose primitive factors are artinian. Then $M_{n}(R)$ satisfies Goodearl-Menal condition for all $n \geq 3$.

Proof. Let $S=M_{n}(R / J(R))(n \geq 3)$. Then $S$ is an exchange ring with all primitive factors artinain. If there exists an ideal $I$ of $S$ such that $S / I \cong \mathbb{Z} / 2 \mathbb{Z}, \mathbb{Z} / 3 \mathbb{Z}, M_{2}(\mathbb{Z} / 2 \mathbb{Z})$, then we have an ideal $K / J(R)$ of $R / J(R)$ such that $M_{n}(R / K) \cong \mathbb{Z} / 2 \mathbb{Z}, \mathbb{Z} / 3 \mathbb{Z}, M_{2}(\mathbb{Z} / 2 \mathbb{Z})$. As $\left|M_{n}(R / K)\right| \geq 2^{n^{2}} \geq 512$, $M_{n}(R / K) \supsetneqq \mathbb{Z} / 2 \mathbb{Z}, \mathbb{Z} / 3 \mathbb{Z}, M_{2}(\mathbb{Z} / 2 \mathbb{Z})$. Hence, $S$ has no homomorphic images $\mathbb{Z} / 2 \mathbb{Z}, \mathbb{Z} / 3 \mathbb{Z}, M_{2}(\mathbb{Z} / 2 \mathbb{Z})$. According to Theorem 3.2, $S$ satisfies GoodearlMenal condition. Clearly, $S \cong M_{n}(R) / J\left(M_{n}(R)\right)$. From this, we deduce that $M_{n}(R)$ satisfies Goodearl-Menal condition, as asserted.

Corollary 3.10. Let $R$ be a semilocal ring. Then $M_{n}(R)$ satisfies Goodearl-Menal condition for all $n \geq 3$.

Proof. Since $R$ is semilocal, $R / J(R)$ is an exchange ring with all primitive factors artinian. The result follows from Proposition 3.9. 
If $G$ is a group and $[G, G]$ its commutator subgroup, then $G^{a b}$ stands for $G /[G, G]$. If $R$ satisfies Goodearl-Menal condition, then $K_{1}(R) \cong U(R)^{a b}$. Let $R=M_{2}(\mathbb{Z} / 2 \mathbb{Z})$. We note that $K_{1}(R) \nsubseteq U(R)^{a b}$. Clearly, $K_{1}(R) \cong$ $\mathbb{Z} / 2 \mathbb{Z} \cong\{1\}$. It is easy to verify that

$$
\begin{aligned}
& U(R)=\left\{\left(\begin{array}{ll}
0 & 1 \\
1 & 0
\end{array}\right),\left(\begin{array}{ll}
0 & 1 \\
1 & 1
\end{array}\right),\left(\begin{array}{ll}
1 & 0 \\
0 & 1
\end{array}\right),\left(\begin{array}{ll}
1 & 0 \\
1 & 1
\end{array}\right),\left(\begin{array}{ll}
1 & 1 \\
0 & 1
\end{array}\right),\left(\begin{array}{ll}
1 & 1 \\
1 & 0
\end{array}\right)\right\}, \\
& {[U(R), U(R)]=\left\{\left(\begin{array}{ll}
1 & 0 \\
0 & 1
\end{array}\right),\left(\begin{array}{ll}
1 & 1 \\
1 & 0
\end{array}\right),\left(\begin{array}{ll}
0 & 1 \\
1 & 1
\end{array}\right)\right\} .}
\end{aligned}
$$

Thus, we see that $\left|U(R)^{a b}\right|=2$, and so $K_{1}(R) \nsubseteq U(R)^{a b}$. But $K_{1}(R) \cong$ $U(R)^{a b}$ if $R=M_{n}(\mathbb{Z} / 2 \mathbb{Z})(n \geq 3)$. In general, $K_{1}(R) \cong G L_{n}(R)^{a b}(n \geq$ 3 ) if $R / J(R)$ is an exchange ring with primitive factors artinian, e.g., $R$ is semilocal. This is an immediate consequence of Proposition 3.9.

Let $S(R)$ be the nonempty set of all ideals of a ring $R$ generated by central idempotents. By Zorn's Lemma, $S(R)$ contains maximal elements. If $P$ is a maximal element of the set $S(R)$, we say that $R / P$ is a Pierce stalk of $R$.

THEOREM 3.11. Let $R$ be an exchange ring whose Pierce stalks are of bounded index. Then $R$ satisfies Goodearl-Menal condition if, and only if it does not admit $\mathbb{Z} / 2 \mathbb{Z}, \mathbb{Z} / 3 \mathbb{Z}, M_{2}(\mathbb{Z} / 2 \mathbb{Z})$ as homomorphic images.

Proof. One direction is clear. Conversely, letting $x, y \in R$. Let

$$
\begin{aligned}
& f_{1}\left(X_{1}, Y_{1}, X_{2}, Y_{2}, X_{3}, Y_{3}\right)=1-\left(X_{1}-X_{2}\right) X_{3}, \\
& f_{2}\left(X_{1}, Y_{1}, X_{2}, Y_{2}, X_{3}, Y_{3}\right)=1-X_{3}\left(X_{1}-X_{2}\right), \\
& f_{3}\left(X_{1}, Y_{1}, X_{2}, Y_{2}, X_{3}, Y_{3}\right)=1-\left(Y_{1}-Y_{2}\right) Y_{3}, \\
& f_{4}\left(X_{1}, Y_{1}, X_{2}, Y_{2}, X_{3}, Y_{3}\right)=1-Y_{3}\left(Y_{1}-Y_{2}\right), \\
& f_{5}\left(X_{1}, Y_{1}, X_{2}, Y_{2}, X_{3}, Y_{3}\right)=1-X_{2} Y_{2}, \\
& f_{6}\left(X_{1}, Y_{1}, X_{2}, Y_{2}, X_{3}, Y_{3}\right)=1-Y_{2} X_{2}
\end{aligned}
$$

be the polynomials in noncommutative indeterminate $X_{1}, Y_{1}, X_{2}, Y_{2}, X_{3}, Y_{3}$. Let $R / P$ be an arbitrary Pierce stalk of $R$. Then $R / P$ is an exchange ring of bounded index. This implies that $R / P$ is an exchange ring with all primitive factors artinian. It is easy to check that $(R / P) / J(R / P)$ is an exchange ring whose primitive factors are artinian. By hypothesis, it is not easy to show that $R / P$ does not admit $\mathbb{Z} / 2 \mathbb{Z}, \mathbb{Z} / 3 \mathbb{Z}, M_{2}(\mathbb{Z} / 2 \mathbb{Z})$ as homomorphic images. According to Theorem 3.2, $R / P$ satisfies Goodearl-Menal condition. Thus, we can find a $u \in U(R / P)$ such that $\bar{x}-u, \bar{y}-u^{-1} \in U(R / P)$. Set $v=u^{-1}$. Then we have some $s, t, c, d \in R / P$ such that

$$
\begin{aligned}
& 1-(\bar{x}-u) s=0, \quad 1-s(\bar{x}-u)=0, \quad 1-(\bar{y}-v) t=0, \\
& 1-t(\bar{y}-v)=0, \quad 1-u v=0, \quad 1-v u=0 .
\end{aligned}
$$


This means that

$$
\begin{aligned}
& f_{1}(\bar{x}, \bar{y}, u, v, s, t)=1-(x-u) s, \\
& f_{2}(\bar{x}, \bar{y}, u, v, s, t)=1-s(x-u), \\
& f_{3}(\bar{x}, \bar{y}, u, v, s, t)=1-(\bar{y}-v) t, \\
& f_{4}(\bar{x}, \bar{y}, u, v, s, t)=1-t(\bar{y}-v), \\
& f_{5}(\bar{x}, \bar{y}, u, v, s, t)=1-u v, \\
& f_{6}(\bar{x}, \bar{y}, u, v, s, t)=1-v u .
\end{aligned}
$$

In view of [12, Lemma 11.4], there exist some $\alpha, \beta, \gamma, \delta \in R$ such that each $f_{i}(x, y, \alpha, \beta, \gamma, \delta)=0$. As a result, we deduce that $x-\gamma, y-\gamma^{-1} \in U(R)$. Therefore $R$ satisfies Goodearl-Menal condition.

Proposition 3.12. Let $R$ be a exchange ring ring whose Pierce stalks are right (left) quasi-duo. Then $R$ satisfies Goodearl-Menal condition if, and only if it does not admit $\mathbb{Z} / 2 \mathbb{Z}, \mathbb{Z} / 3 \mathbb{Z}, M_{2}(\mathbb{Z} / 2 \mathbb{Z})$ as homomorphic images.

Proof. Let $x, y \in R$. Construct the polynomials $f_{1}, \cdots, f_{6}$ as in Theorem 3.11. Let $R / P$ be an arbitrary Pierce stalk of $R$. Then $R / P$ is a right (left) quasi-duo exchange ring. In view of Corollary 3.8, $R / P$ satisfies Goodearl-Menal condition. As in the proof of Theorem 3.11, there exist some $\alpha, \beta, \gamma, \delta \in R$ such that each $f_{i}(x, y, \alpha, \beta, \gamma, \delta)=0$. Consequently, $x-\gamma, y-\gamma^{-1} \in U(R)$, as required

A commutative ring $R$ satisfies the primitive criterion if for each polynomial $f(x)=a_{0}+a_{1} x+\cdots+a_{n} x^{n}(n \geq 0)$ with $a_{0} R+\cdots+a_{n} R=R$, i.e., $f(x) \in R[x]$ is primitive, then there exists an $\alpha \in R$ such that $f(\alpha) \in U(R)$ (cf. [8]). As is well known, every commutative ring satisfying the primitive criterion satisfies Goodearl-Menal condition. If $R / J(R)$ is a commutative exchange ring, it follows that $R$ satisfies Goodearl-Menal condition if, and only if $|R / M| \geq 4$ for all maximal ideals $M$ of $R$. Explicitly, we can derive the following.

Proposition 3.13. Let $R / J(R)$ be a commutative exchange ring. Then the following are equivalent:

(1) $R$ satisfies the primitive criterion.

(2) $R / M$ is an infinite field for all maximal ideals $M$ of $R$.

Proof. (1) $\Rightarrow(2)$ Suppose that $R$ satisfies the primitive criterion and $M$ is a maximal ideal of $R$. Then $R / M$ is a field. Assume that $R / M=$ $\left\{\overline{x_{1}}, \cdots, \overline{x_{n}}\right\}$ is a finite field. Let $f(x)=\left(x-x_{1}\right) \cdots\left(x-x_{n}\right) \in R[x]$. Then $f(x)$ is primitive; hence, there exists some $\alpha \in R$ such that $f(\alpha) \in U(R)$. This implies that $\bar{f}(\bar{\alpha})=\left(\bar{\alpha}-\overline{x_{1}}\right) \cdots\left(\bar{\alpha}-\overline{x_{n}}\right) \in U(R / M)$, and so $\bar{\alpha} \notin R / M$. This gives a contradiction. Therefore $R / M$ is an infinite field.

$(2) \Rightarrow(1)$ If $R / J(R)$ satisfies the primitive criterion, then so does $R$. Thus, without loss of the generality, we may assume that $R$ is a commutative 
exchange ring. Assume that $R$ doesn't satisfy the primitive criterion. Then there exists a primitive $f(x)=a_{0}+a_{1} x+\cdots+a_{n} x^{n}$ such that $f(\alpha) \notin U(R)$ for all $\alpha \in R$. Let $\Omega$ be the set of all the ideals $A$ of $R$ such that $\bar{f}(\bar{\alpha})=$ $\overline{a_{0}}+\overline{a_{1} \alpha}+\cdots+\overline{a_{n} \alpha^{n}} \notin U(R / A)$ for all $\alpha \in R$. Clearly, $\Omega \neq \emptyset$.

Given any ascending chain $A_{1} \subseteq A_{2} \subseteq \cdots \subseteq A_{k} \subseteq \cdots$ in $\Omega$, we set $M=\bigcup_{i=1}^{\infty} A_{i}$. Then $M$ is an ideal of $R$. If $M$ is not in $\Omega$, then there exists $\alpha \in R$ such that $\bar{f}(\bar{\alpha}) \in U(R / M)$. Hence, we have some $r \in R$ such that $f(\alpha) r-1 \in M$. Thus, we can find positive integers $n$ such that $f(\alpha) r-1 \in A_{n}$; hence, $\bar{f}(\bar{\alpha}) \in U\left(R / A_{n}\right)$. This gives a contradiction. Thus, $\Omega$ is inductive. By using Zorn's Lemma, we have an ideal $Q$ of $R$ such that $Q$ is maximal in $\Omega$. Let $S=R / Q$. The maximality of $Q \in \Omega$ implies that $S$ is indecomposable as a ring. If $J(S) \neq 0$, we may assume that $J(S)=N / Q$ with $Q \varsubsetneqq N$. By the maximality of $Q$, there exists some $\alpha \in R$ such that $\bar{f}(\bar{\alpha}) \in U(R / N)$. Since $S / J(S) \cong R / N$, we may assume that $\bar{f}(\bar{\alpha}) \in U(S / J(S))$. As units lift modulo the Jacobson radical of $S$, we see that $\bar{f}(\bar{\alpha}) \in U(R / Q)$, and yields a contradiction. This implies that $J(S)=0$, so $S$ is an indecomposable ring with $J(S)=0$. Since $R$ is a commutative exchange ring, $S$ is simple artinian. That is, $S$ is a field. We infer that $Q$ is a maximal ideal of $R$, and so $R / Q$ is an infinite field. Thus, we can find some $\beta \in R$ such that $\bar{f}(\bar{\beta}) \in U(R / Q)$, a contradiction. Therefore $R$ satisfies the primitive criterion.

Corollary 3.14. Let $R$ be a commutative exchange ring. Then the following are equivalent:

(1) $R$ satisfies the primitive criterion.

(2) $R / M$ is an infinite field for all maximal ideals $M$ of $R$.

Proof. In view of [12, Theorem 29.2], $R / J(R)$ is an exchange ring. Therefore we complete the proof by Proposition 3.13 .

As an immediate consequence, we claim that $R$ satisfies the primitive criterion if and only if $R / M$ is an infinite field for all maximal ideals $M$ of $R$ if $R$ is generalized $n$-like $(n \geq 2)$, i.e., $(x y)^{n}-x y^{n}-x^{n} y+x y=0$ for any $x, y \in R$. In this case, $R / J(R)$ is a commutative exchange ring, and we are done.

ACKNOWLEDGEMENTS.

The author is grateful to the referee for his/her suggestions which correct several errors in the first version and make the new one clearer.

\section{REFERENCES}

[1] H. Chen, Exchange rings with Artinian primitive factors, Algebr. Represent. Theory 2 (1999), 201-207.

[2] H. Chen, Exchange rings satisfying unit 1-stable range, Kyushu J. Math. 54 (2000), $1-6$. 
[3] H. Chen, Units, idempotents, and stable range conditions, Comm. Algebra 29 (2001), 703-717.

[4] H. Chen, Decompositions of countable linear transformations, Glasg. Math. J. 52 (2010), 427-433

[5] L. Wang and Y. Zhou, Decompositions of linear transformations, Bull. Aust. Math. Soc., to appear.

[6] K. R. Goodearl and P. Menal, Stable range one for rings with many units, J. Pure Appl. Algebra 54 (1988), 261-287.

[7] C. Huh, N. K. Kim and Y. Lee, On exchange rings with primitive factor rings Artinian, Comm. Algebra 28 (2000), 4989-4993.

[8] B. R. McDonald, Projectivities over rings with many units, Comm. Algebra 9 (1981), 195-204.

[9] P. Menal, On $\pi$-regular rings whose primitive factor rings are artinian, J.Pure Appl. Algebra 20 (1981), 71-78.

[10] F. Perera, Lifting units modulo exchange ideals and $C^{*}$-algebras with real rank zero, J. Reine Angew. Math. 522 (2000), 51-62.

[11] J. Rosenberg, Algebraic $K$-theory and its applications, Springer-Verlag, New York, 1994.

[12] A. A. Tuganbaev, Rings close to regular, Kluwer Academic Publishers, Dordrecht, 2002.

[13] H. P. Yu, Stable range one for exchange rings, J. Pure Appl. Algebra 98 (1995), 105-109.

[14] H. P. Yu, On the structure of exchange rings, Comm. Algebra 25 (1997), 661-670.

H. Chen

Department of Mathematics

Hangzhou Normal University

Hangzhou 310036

China

E-mail: huanyinchen@yahoo.cn

Received: 21.3 .2011$.

Revised: 9.8.2011. \& 25.10.2011. 